\title{
Accurate global ab initio potentials at low-cost by correlation scaling and extrapolation to the one-electron basis set limit
}

\author{
A.J.C. Varandas * \\ Departamento de Química, Universidade de Coimbra 3004-535 Coimbra, Portugal \\ Received 16 April 2007; in final form 24 May 2007 \\ Available online 17 June 2007
}

\begin{abstract}
A recently proposed scheme that enables a potential energy surface corresponding to a calculation with a large target basis set to be obtained from small basis set calculations via scaling of the electron correlation at a single-pivotal geometry is generalized to include an arbitrary number of such geometries. If the correlation is extrapolated to the complete basis set limit at the pivotal geometries and used for the scaling, the method can yield accurate potentials at costs up to factors nearly fifty times cheaper than required otherwise. The approach, free from parameters alien to the ab initio methods, is tested on $\mathrm{N}_{2}, \mathrm{O}_{2}$, and $\mathrm{F}_{2}$.
\end{abstract}

(C) 2007 Elsevier B.V. All rights reserved.

\section{Introduction}

The cost of solving the electronic Schrödinger equation point-by-point to generate a potential energy surface (PES) is well known to grow as $N_{\mathrm{p}}^{\tau} n_{\mathrm{b}}^{m}$ with $m \gtrsim 4$, where $n_{\mathrm{b}}$ is the number of orbitals in the basis set, and $N_{\mathrm{p}}$ a typical number of points required to represent a one-dimensional (1D) cut in the $\tau D$ configuration space of the molecule. Of course, many such 'diatomic-like' cuts will be required to map the PES of a polyatomic molecule, e.g., one for each fixed set of Jacobi angles. An even stronger factorial scaling of the cost with the number of active electrons $\left(n_{\mathrm{e}}\right)$ arises if the multi-reference configuration interaction (MRCI) method which is based on the preceding complete-activespace self-consistent-field (CASSCF or CAS for brevity) approach is utilized as for a fixed number of electrons the number of determinants in a full CI (the CAS wave function is a FCI in the active orbital space) scales [1] as $n_{\mathrm{b}}^{n_{\mathrm{e}}}$. Obtaining an accurate PES with as low as possible computational cost remains therefore an important challenge in quantum chemistry. Without any lack of generality, the 'economical' approach here suggested will be tested on the important $\mathrm{N}_{2}$,

\footnotetext{
${ }^{*}$ Fax: +351239835867.

E-mail address: varandas@qtvs1.qui.uc.pt.
}

$\mathrm{O}_{2}$, and $\mathrm{F}_{2}$ molecules. The highly successful internally contracted MRCI approach with the popular quasi-degenerate Davidson correction $[\mathrm{CAS} / \mathrm{MRCI}(Q)$ or simply $\operatorname{MRCI}(Q)]$ $[2,3]$ will be employed for the calculations.

A significant enhancement to progress in ab initio calculations for small molecules has become possible after the introduction of the so-called correlation-consistent (cc) basis sets by Dunning and collaborators [4] such as the augmented one aug-cc-pV $X \mathrm{Z}$ [5] (or AV $X \mathrm{Z}$ ). Built in a systematic manner intended to relate the correlation energy to the cardinal number $X$, such basis sets prompted the search for laws to extrapolate the energy to the complete basis set (CBS) limit (e.g., Refs. [6-10]).

The extrapolation of the correlation energy finds support on its dependence on the partial wave quantum number for two-electron atomic systems and second-order pair energies in many-electron atoms $[11,12]$. The simplest and most popular two-parameter law for extrapolation of the correlation energy is [6]

$E_{X}^{\mathrm{cor}}=E_{\infty}^{\mathrm{cor}}+\frac{A_{3}}{(X+\alpha)^{3}}$

where $E_{X}^{\text {cor }}$ is the correlation energy obtained with the $X$-tuple basis set of (aug)cc quality, and $E_{\infty}^{\text {cor }}$ and $A_{3}$ are parameters usually determined from the calculations for 
the two highest affordable values of $X$, often 5 and $6 ; \alpha$ is an offset parameter fixed from some auxiliary condition.

Generalized three-parameter extrapolation rules have also been suggested

$E_{X}=E_{\infty}+\frac{A_{3}}{(X+\alpha)^{3}}+\frac{A_{n}}{(X+\alpha)^{n}}$

In particular, by choosing $A_{n}$, with $n=4$, to depend on $A_{3}$, we have suggested [8] an effective two-parameter rule that predicts the estimated CBS correlation energy limit with a root mean squared deviation (rmsd) of only a few millihartree for a set of 33 systems studied by the popular single-reference Møller-Plesset (MP2), and coupled cluster [CCSD and CCSD(T)] methods. Recently, we have shown that both the full correlation and its dynamical part can be rather accurately described via a refinement of the above law, termed as uniform singlet- and triplet-pair extrapolation (USTE [10]) method. With $n=5$ and $\alpha=3 / 8$, the auxiliary relation assumes the form [10]

$A_{5}=A_{5}(0)+c A_{3}^{5 / 4}$

where $A_{5}(0)=0.0037685459$ and $c=-1.17847713 E_{h}^{-5 / 4}$ for the dynamical correlation.

For the uncorrelated Hartree-Fock and CAS (uncorrelated in the sense of lacking dynamical correlation) energies, several schemes have been advanced (Refs. [13,10], and references therein). We will use the form

$E_{X}^{\mathrm{CAS}}=E_{\infty}^{\mathrm{CAS}}+A \exp (-b X)$

where $E_{\infty}^{\mathrm{CAS}}, A$, and $b$ are parameters to be determined from a fit to the raw CAS energies, typically the ones calculated for the three or more highest affordable cardinal numbers. Such extrapolations are known to attain a high accuracy when raw energies up to $X=6$ are utilized, and we utilize one such variant [10]. The situation is less clear when only raw data up to $X=Q$ is available, although we will consider later also the two-point extrapolation formula proposed by Karton and Martin [13], $A+B / X^{5.34}$.

Note that the extrapolation of $E_{X}^{\mathrm{CAS}}$ and $E_{X}^{\mathrm{cor}}$ with $X$ is geometry-dependent, and hence such a procedure for calculating a molecular PES must be carried out pointwise. This can be a mammoth task even for the fastest computers if the raw data requires basis sets with large cardinal numbers. Although the USTE $[10,14]$ scheme has shown great promise in extrapolating from $(T, Q)$ cardinal number pairs, we explore in this work ways for a further drastic reduction in labor by obtaining the dynamical correlation at the $\operatorname{MRCI}(Q) / \operatorname{AV} Q Z$ basis set level via a correlation scaling (CS) procedure recently suggested by Varandas and Piecuch [15]. This is based on scaling at a single geometry in configuration space (hereby called as pivotal geometry or simply pivot) the difference between the total energy $E$ and the energy of some reference wave function, $E^{\text {ref }}$, which represents the correlation energy or part of it. In the case of the $\operatorname{MRCI}(Q)$ method here employed, the reference wave function is obtained in the preceding CAS calculations (see Ref. [15] for alternative choices using other quantum methods).

The approach here suggested involves six steps: (a) calculation of the PES at, say $N_{\mathrm{p}}$ points, with the $X-2$ and $X-1$ basis sets using $\operatorname{MRCI}(Q)$, and $N=1-4$ such calculations with the target basis set of rank $X$ at some pre-chosen pivots (see later); (b) $N_{\mathrm{p}}-N$ calculations of the CAS (here taken as the reference) PES at the target $X$ basis set level; (c) prediction by extrapolation $[10,13]$ to $X=\infty$ of the CAS/CBS PES; (d) prediction by extrapolation to $X=\infty$ of the dynamical correlation energy at the pivots by using the USTE/(T,Q) method [10]; (e) prediction of the CBS dynamical correlation at the remaining $N_{\mathrm{p}}-N$ points by CS using the $X=T$ (or $X=D$ ) and the CBS dynamical correlation energies at the $N$ pivots; (f) calculation of the full CBS PES by adding the CAS/CBS and extrapolated dynamical correlation energies from steps (c) and $(\mathrm{d}, \mathrm{e})$. For brevity such CAS/CBS $+\mathrm{CS}^{N} / \mathrm{USTE}$ energies will be denoted heretofore without apparent confusion by $\mathrm{CS}^{N} /$ USTE. For specificity, we indicate after the acronym USTE the cardinal number pair associated to the raw dynamical correlation energies utilized for the extrapolation.

In the following, we will consider $\mathrm{AVQZ}$ as an expensive basis set, as it is perhaps the largest affordable basis for small molecules. Thus, except for the $\operatorname{MRCI}(Q) / \mathrm{AV} Q \mathrm{Z}$ calculations at $N=1-4$ pivotal geometries, the bulk calculations employ only the modest AVDZ and AVTZ basis sets. The quality of the $\operatorname{CS}^{N} / \operatorname{USTE}(T, Q)$ potentials so obtained will then be tested through vibrational calculations, which provide a most severe test of the method as the results depend on relative energies. The Letter is organized as follows. In Section 2, we describe the correlation scaling-extrapolation procedure to obtain the PES at the target basis set, while the results are reported and discussed in Section 3. The concluding remarks are in Section 4.

\section{Generalized correlation scaling-extrapolation method}

Let without any lack of generality from the computational point of view consider a PES as a bunch of $1 \mathrm{D}$ cuts through its configuration space. Let the appropriate coordinate (radial or angular or any suitable combination of both) defining the one-dimensional domain along such a cut be denoted as $R$. Suppose now that $\operatorname{MRCI}(Q)$ or some other correlated calculations using basis sets indexed by $m-k$ and $m$ are performed $(m \equiv X$ for $\mathrm{AV} X \mathrm{Z}$, but it can be an arbitrary index for basis sets that do not belong to the cc family [15]) within such a domain. The extrapolated $\operatorname{MRCI}(Q)$ for the target $(m+\ell)$ th basis set will be obtained from

$E_{m+\ell}(R)=E_{m+\ell}^{\mathrm{ref}}(R)+\left[1+\mathscr{P}_{m+\ell, m}^{(N)}(R)\right] \Delta E_{m}^{\mathrm{cor}}(R)$

where a first choice for $\mathscr{P}_{m+\ell, m}^{(N)}(R)$ would be an interpolating polynomial of degree $N-1$ through $N$ points $\left\{y_{i}=y\left(R_{i}\right)\right\}$ as given by the Lagrange classical formula 


$$
\begin{aligned}
\mathscr{P}_{m+\ell, m}^{(N)}(R)= & \frac{\left(R-R_{2}\right)\left(R-R_{3}\right) \cdots\left(R-R_{N}\right)}{\left(R_{1}-R_{2}\right)\left(R_{1}-R_{3}\right) \cdots\left(R_{1}-R_{N}\right)} y_{1} \\
& +\frac{\left(R-R_{1}\right)\left(R-R_{3}\right) \cdots\left(R-R_{N}\right)}{\left(R_{2}-R_{1}\right)\left(R_{2}-R_{3}\right) \cdots\left(R_{2}-R_{N-1}\right)} y_{2} \\
& +\cdots \\
& +\frac{\left(R-R_{1}\right)\left(R-R_{2}\right) \cdots\left(R-R_{N-1}\right)}{\left(R_{N}-R_{1}\right)\left(R_{N}-R_{2}\right) \cdots\left(R_{N}-R_{N-1}\right)} y_{N}
\end{aligned}
$$

In turn, the unitless points to be interpolated are defined by

$y(R)=\frac{\left[S(R)_{m, m-k}-1\right]\left[S\left(R_{i}\right)_{m+\ell, m}-1\right]}{S\left(R_{i}\right)_{m, m-k}-1}$

where the index $i$ specifies a pivotal geometry where the scaling is performed, $k$ and $\ell$ are suitable positive integers, and the auxiliary function $S(R)$ assumes the form

$S(R)_{m, m-k}=\Delta E_{m}^{\mathrm{cor}}(R) / \Delta E_{m-k}^{\mathrm{cor}}(R)$

with $E_{m-k}^{\text {ref }}(R)$ being the energy of the reference wave function (here taken as the CAS wave function) for the $m-k$ basis set, and $\Delta E_{m-k}^{\mathrm{cor}}(R)=E_{m-k}(R)-E_{m-k}^{\mathrm{ref}}(R)$ the associated dynamical correlation energy. Corresponding definitions apply to $\Delta E_{m}^{\mathrm{cor}}(R)$ and $E_{m+\ell}^{\mathrm{ref}}(R)$ in the above equations, with the latter being the energy of the reference wave function calculated with the $(m+\ell)$ th target basis set. Thus, if there are $N$ energies calculated with the $(m+\ell)$ th basis set, there will be $N$ terms in Eq. (6), each a polynomial of degree $(N-1)$ and each constructed to be zero at all $R_{i}$ values but one where it is here constructed to be $y_{i}=y\left(R_{i}\right)$. In fact, Eq. (5) is perhaps the simplest mathematical expression that one can suggest to interpolate the energies based on scaling the correlation energy (or part of it if other wave function model is employed) from basis set $m$ to basis set $m+\ell$ at the pivots. In particular, the scaling function $\left[1+\mathscr{P}_{m+\ell, m}^{(N)}(R)\right]$ in Eq. (5) satisfies the following attributes: (i) $\left[1+\mathscr{P}_{m+\ell, m}^{(N)}(R)\right] \rightarrow 1$ for all values of $R$ when $m \rightarrow \infty \quad$ as $y(R) \rightarrow 0$ due to $S(R)_{m, m-k} \rightarrow 1$ and $S(R)_{m+\ell, m} \rightarrow 1$, and the fact that the numerator of Eq. (7) vanishes faster than the denominator; (ii) the value of $\left[1+\mathscr{P}_{m+\ell, m}^{(N)}(R)\right]$ at any of the pivots equals the ratio of $\Delta E_{m+\ell}^{\text {cor }}$ and $\Delta E_{m}^{\text {cor }}$. If $N=1$, the method corresponds to the scheme proposed elsewhere [15]. In this case, there is a single pivot which can be the equilibrium geometry $R_{\mathrm{e}}$ or any other point on the PES. Thus, the $\operatorname{MRCI}(Q)$ or some other PES $E_{m+\ell}(R)$ is extrapolated from the PESs $E_{m}(R)$ and $E_{m-k}(R)$, the corresponding reference energies $E_{m+\ell}^{\mathrm{ref}}(R), E_{m}^{\mathrm{ref}}(R)$ and $E_{m-k}^{\mathrm{ref}}(R)$, and $N$ correlated energies calculated at the selected pivots (reference geometries) $R_{1}, R_{2}, \ldots, R_{N}$, namely $E_{m+\ell}\left(R_{1}\right), E_{m+\ell}\left(R_{2}\right), \ldots, E_{m+\ell}\left(R_{N}\right)$.

Although mathematically rigorous, the Lagrange formalism may yield artifacts outside the range of interpolated data, particularly when high-order polynomials are used in the interpolation procedure. We have therefore tested also a procedure based on switching forms. Although tanh functions are most popular for this purpose, we employ more flexible forms that warrant continuity up to a prechosen order $\tilde{m}-1$ in the derivatives, where $\tilde{m}$ is a positive number greater than 1 , here taken as 2 . For the two-pivot case (partition of the $R$-domain for $R \leqslant R_{\text {out }}$ in two sectors: one characterizing regions up to the first pivot, $R_{\text {ref }}$, the other characterizing the outer part, $R>R_{\text {ref }}$, one may write:

$$
\begin{aligned}
& y(R)=y_{\text {ref }}+\Delta y_{\text {out }}\left\{1-\exp \left[-\beta\left(R-R_{\text {ref }}\right)^{\tilde{m}}\right]\right\} \quad R \geqslant R_{\text {ref }} \\
& y(R)=y_{\text {ref }} \quad R<R_{\text {ref }}
\end{aligned}
$$

where $\Delta y_{\text {out }}=y_{\text {out }}-y_{\text {ref }}$, and $\beta$ is chosen such that $y\left(R_{\text {out }}\right) \sim y_{\text {out }}$ within an acceptable tolerance (typically $0.1 \%)$. For example, a typical value of $\beta$ for $\mathrm{N}_{2}$ is $1.5 \AA^{-1}$, although other values close to it could work equally well or perhaps slightly better. Note that $y_{\text {out }}$ is the value obtained from Eq. (7) at the pivot $R_{\text {out }}$, with corresponding definitions applying for the other $y_{i}$ values. Note further that $R_{\text {ref }}$ has been taken as the experimental equilibrium geometry, and $R_{\text {out }}$ as a geometry sufficiently far from equilibrium to represent the limiting behavior. Thus, for a diatomic, the above switching function can impose a scaling trend such as to warrant reproduction of the correct energy at the dissociation asymptote. For the threesector case where there are an inner $\left(R_{\mathrm{inn}}\right)$, an intermediate $\left(R_{\text {ref }}\right)$, and an outer $\left(R_{\text {out }}\right)$ pivots, we may write:

$$
\begin{aligned}
& y(R)=y_{\text {inn }} \quad R \leqslant R_{\text {inn }} \\
& y(R)=y_{\text {ref }}+\Delta y_{\text {inn }}\left\{1-\exp \left[-\beta_{\text {inn }}\left(R-R_{\text {ref }}\right)^{\tilde{m}}\right]\right\} \\
& R_{\text {inn }} \leqslant R \leqslant R_{\text {ref }} \\
& y(R)=y_{\text {ref }}+\Delta y_{\text {out }}\left\{1-\exp \left[-\beta_{\text {out }}\left(R-R_{\text {ref }}\right)^{\tilde{m}}\right]\right\} \\
& \quad R>R_{\text {ref }}
\end{aligned}
$$

where $\Delta y_{\text {inn }}=y_{\text {inn }}-y_{\text {ref }}, \Delta y_{\text {out }}=y_{\text {out }}-y_{\text {ref }}$, and $\beta_{\text {inn }}$ and $\beta_{\text {out }}$ are parameters chosen as above to satisfy the boundary conditions. For example, for $\mathrm{N}_{2}$, typical $\beta$ values are $\beta_{\text {inn }}=55.0 \AA^{-1}$ and $\beta_{\text {out }}=1.5 \AA^{-1}$. Of course, if the innermost and outermost pivots ( $y_{\text {inn }}$ and $y_{\text {out }}$ ) correspond to the extreme calculated points along the $R$-coordinate, only Eqs. (12) and (13) apply. Forms with more pivots can similarly be developed.

The ability of the CS method to predict the PES corresponding to larger basis sets from smaller basis set calculations with sub-millihartree accuracies suggests that the procedure can be combined with available schemes to extrapolate the PES to the CBS limit. Two distinct approaches can be followed. The first consists of treating the extrapolated $\operatorname{USTE}(T, Q)$ energies at the pivots as $E_{m+\ell}\left(R_{i}\right)(i=1-N)$, and apply the above CS method. Alternatively, one could extrapolate at every point to $X=\infty$ using the raw AVTZ energies and the AVQZ ones estimated via the $\operatorname{USTE}(T, Q)$ scheme (or else the raw $\operatorname{MRCI}(Q) / \operatorname{AV} Q Z$ energies at the pivots). Since the fit of a $\tilde{n}$-parameter law to $\tilde{n}$-points is an ill-posed problem in the sense that in no way the procedure can be tested (i.e., relies on the exactness of both the numerical data and underlying physical law), we will adopt here the first scheme. If the other had been chosen, it might be advisable to employ instead the $\operatorname{USTE}(D-Q)$ extrapolation scheme 
[10] as this is expected to be stabilized by the use of three fitted points. The method is 'analytic' to the order imposed by the power $\tilde{m}$ in Eqs. (9)-(13) at one pivot, here chosen as $R_{\text {ref }}$, and up to the allowed matching tolerance at the other pivots. Although this can be controlled such that the boundary conditions are matched to a desired accuracy, one may wish to avoid extreme cases since they originate abrupt changes in $y(R)$. Indeed, any small discontinuities in $y(R)$ and its derivatives should not be troublesome as the raw $a b$ initio energies and therefore their derivatives exhibit also some inescapable numerical noise. Thus, we may for all purposes consider as smooth the resulting PES, being probably adequate for direct dynamics studies. In fact, any such small errors will likely pass unnoticed if the derivatives are calculated numerically as often done in dynamics. Finally, we emphasize that both the singlepivot and multi-pivot methods (if based on the Lagrange formalism) are analytic, with the latter showing in principle no handicap if extrapolations in $R$ will not occur. In fact, hybrid approaches using both the Lagrange and switching-function formalisms may also be envisaged. We further note that the method has no empirically adjustable parameters, and hence contrasts with other single-pivot schemes [16-18] that extrapolate the correlation energy but choose the scaling to fit some well known experimental attribute of the molecule such as available atomization or dissociation energies.

\section{Results and discussion}

All $\operatorname{MRCI}(Q) / \mathrm{AV} X \mathrm{Z}(X=D, T, Q)$ calculations have been performed using MOLPRO [19]. As test systems, we have chosen $\mathrm{N}_{2}, \mathrm{O}_{2}$, and $\mathrm{F}_{2}$ in their ground electronic states, as these molecules are representative of three of the most frequent types of chemical bonds (single in $\mathrm{F}_{2}$, double in $\mathrm{O}_{2}$, and triple in $\mathrm{N}_{2}$ ). For all species, the active space in the CAS and subsequent $\operatorname{MRCI}(Q)$ calculations consisted of the valence orbitals that correlate with the $2 \mathrm{~s}$ and $2 \mathrm{p}$ atomic shells. The lowest-energy molecular orbitals (involving the $1 s$ orbitals) have been optimized in the CAS calculations, but their occupancies remained doubly occupied in all reference determinants that define the CAS and MRCI wave functions. For comparison purposes, $\operatorname{MRCI}(Q)$ calculations have also been carried out over the same range of geometries using the AV5Z and AV6Z basis sets. Although core electrons may bring significant contributions, core and core-valence correlation effects will therefore be ignored, in addition to relativistic and nonadiabatic correction effects. As indicated by the symbols in Fig. 1, the calculations covered $N_{\mathrm{p}}=29$ geometries over the range of reduced bond distances $0.7 \leqslant R / R_{\mathrm{e}} \leqslant 5.0$, with $R_{\mathrm{e}}$ taken as the experimental equilibrium geometry [20].

Fig. 1 shows the results obtained from both the $(m, m-k ; m+\ell, m)=(3,2 ; 4,2)$ and $(3,2 ; 4,3)$ CS schemes. The top panels illustrate the changes $\Delta E_{m+\ell, m-k}^{\mathrm{cor}}=E_{m+\ell}^{\mathrm{cor}}-$ $E_{m-k}^{\mathrm{cor}}$ in correlation energy when going from the smaller to the target basis set, namely $\Delta E_{42}^{\text {cor }}=E_{4}^{\text {cor }}-E_{2}^{\text {cor }}$ and $\Delta E_{43}^{\text {cor }}=E_{4}^{\text {cor }}-E_{3}^{\text {cor }}$, while the corresponding errors $\epsilon_{m+\ell, m-k}^{\text {cor }}$ are plotted in the bottom panels. As Fig. 1 shows, the target correlation energy is recovered within a millihartree or so accuracy when utilizing a single pivot at $R=R_{\mathrm{e}}$, but this result can be improved by a factor of at least fivefold if additional pivots are utilized. Of course, using more than four pivots would hardly fit the goal of low-cost PESs,
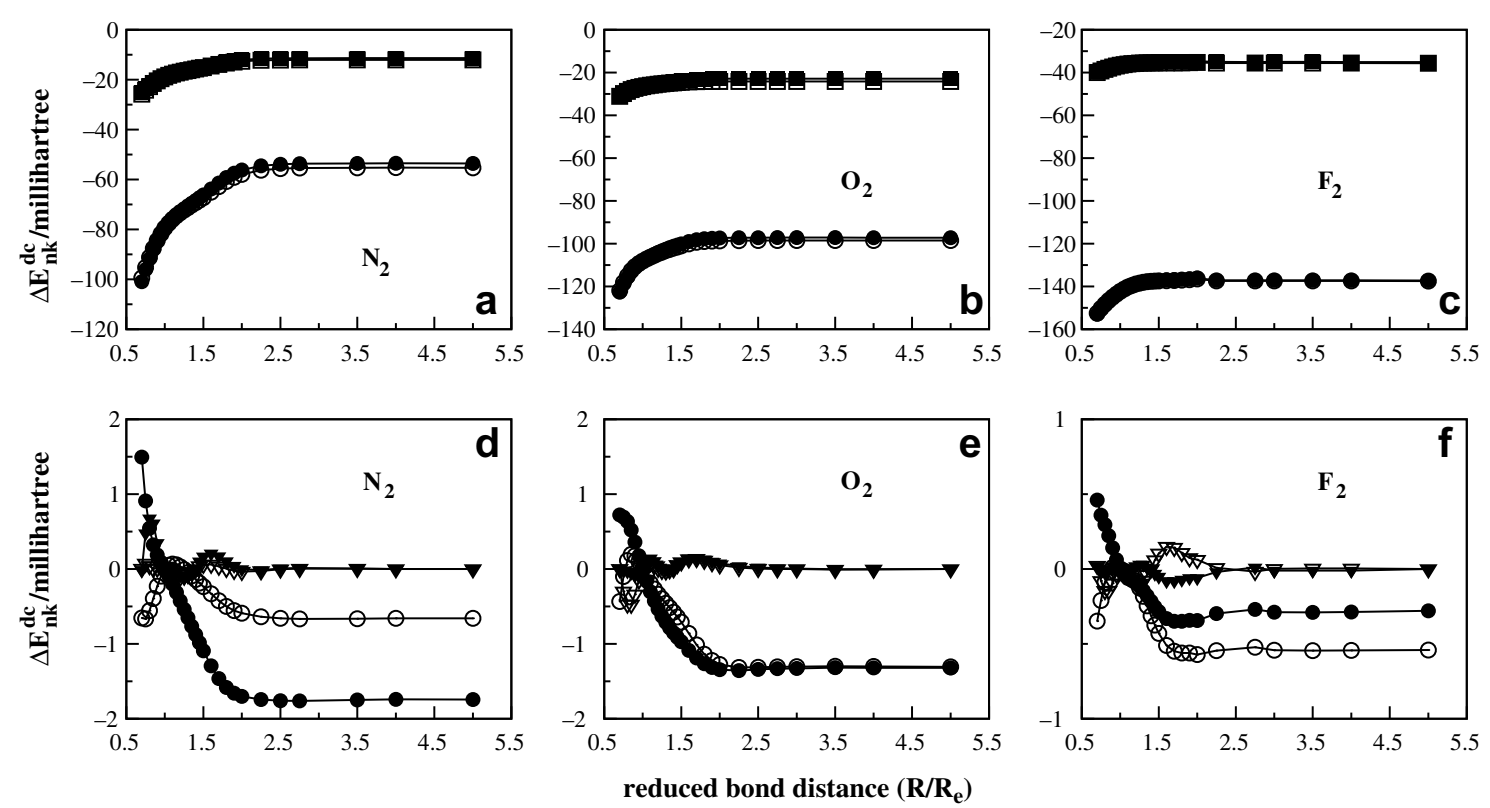

Fig. 1. A comparison of the actual and predicted changes in $\operatorname{MRCI}(Q) / \mathrm{AV} X \mathrm{Z}$ correlation energies when going from basis set $m-k$ to basis set $m+\ell$, $\Delta E_{m+\ell, m-k}=E_{m+\ell}-E_{m-k}$ : (a) $\mathrm{N}_{2}$; (b) $\mathrm{O}_{2}$; (c) $\mathrm{F}_{2}$. The corresponding errors when using 1 and 4 pivots are shown in panels (d)-(f). With $m=3$ and $\ell=1$, the dashed lines are for $k=1$ and the solid ones for $k=0$. The line segments that link the calculated points are drawn to guide the eye, with the points at $R / R_{\mathrm{e}}=3$ for $\mathrm{N}_{2}$ and $R / R_{\mathrm{e}}=2.5$ for $\mathrm{F}_{2}$ removed for clarity as they would originate meaningless spikes. 
and hence it has not been considered. The results are best appreciated from Table 1 where the rmsd between the predicted and raw energies calculated at the target basis set level are collected. For example, in the case of $\mathrm{N}_{2}$ at the $(3,2 ; 4,2) \mathrm{CS}$ level, the rmsd varies from $0.438(0.438)$ for a single pivot [15] to 0.358 (0.243), 0.259 (0.097), and $0.132(0.100) \mathrm{mE}_{\mathrm{h}}$ for scaling at two, three, and four pivots using the Lagrange (switching function) formalism. Clearly, the results depend on the location of the pivots, which could be optimized to reduce the rmsd. Since this may be considered to be expensive, as there is no obvious way of finding the optimum location prior to detailed $a b$ initio calculations, we have excluded such a step for generality, except in what concerns the obvious equilibrium and (the chosen) dissociation geometries. Thus, the pivots have in all cases been fixed at: $R_{\mathrm{e}}$ for the single-pivot $\left(\mathrm{CS}^{1}\right)$ case; $R_{\mathrm{e}}$ and $5 R_{\mathrm{e}}$ for $\mathrm{CS}^{2} ; 0.7 R_{\mathrm{e}}, R_{\mathrm{e}}$, and $5 R_{\mathrm{e}}$ for $\mathrm{CS}^{3} ; 0.7 R_{\mathrm{e}}$, $R_{\mathrm{e}}, 1.4 R_{\mathrm{e}}$, and $5 R_{\mathrm{e}}$ for $\mathrm{CS}^{4}$. Our choice corresponds in $\mathrm{CS}^{2}$ to divide the space into two sectors (up to equilibrium and beyond it, up to the chosen asymptote). For $\mathrm{CS}^{3}$, an additional pivot has been considered at the repulsive wing of the potential (here taken as the shortest calculated bond distance), while in $\mathrm{CS}^{4}$ the outer branch has been subdivided in two parts with the fourth pivot (point \#15) being chosen as the middle point of the 29 that were calculated.

The dependence of the rmsd on the pivotal geometries may explain its non-monotonic decrease with their number. For example, for the $(4,2 ; 4,3)$ scheme using the Lagrange (switching) interpolation procedure, the rmsd for $\mathrm{N}_{2}$ are 1.196 (1.196), 0.262 (0.446), 0.198 (0.341), and 0.253 (0.235) $\mathrm{mE}_{\mathrm{h}}$. Since the predictions for distances shorter than the first pivot and larger than the last can be erratic in the Lagrange approach, only the switching function results will be examined. Note that $\mathrm{CS}^{2}$ suffices to mimic the diatomic atomization energy at the target basis set level. We further observe that slightly smaller errors are generally obtained when the Davidson correction is not included.

Extrapolation to the CBS limit can now be considered by using the extrapolated $\operatorname{USTE}(T, Q)$ correlation energies at the pivots as the results at the target $X=\infty$ basis set. For brevity, we consider only the $(4,3$; USTE, 4$)$ correlation scaling scheme, where USTE stands now for $\operatorname{USTE}(T, Q)$. Note that the error due to the $\operatorname{USTE}(T, Q)$ extrapolation scheme has been shown elsewhere [10] to be rather small when judged from the $\operatorname{USTE}(5,6)$ results. At equilibrium, they amount to [10] $-0.737,-0.572$, and $-0.155 \mathrm{mE}_{\mathrm{h}}$ for $\mathrm{N}_{2}, \mathrm{O}_{2}$, and $\mathrm{F}_{2}$, with similarly good results extending up to dissociation. Once the extrapolated $(4,3$; USTE, 4$)$ dynamical correlation energies have been predicted, the full extrapolated curve can be obtained by adding the reference CAS/CBS energy as predicted by our own rule [10]. This consists of extrapolating the raw CAS energies obtained with the four largest affordable $\operatorname{AVXZ}$ basis sets $(X=T, Q, 5,6)$ with the three-parameter exponential law in Eq. (4), and take as the recommended result the average of the value so obtained with the largest affordable raw

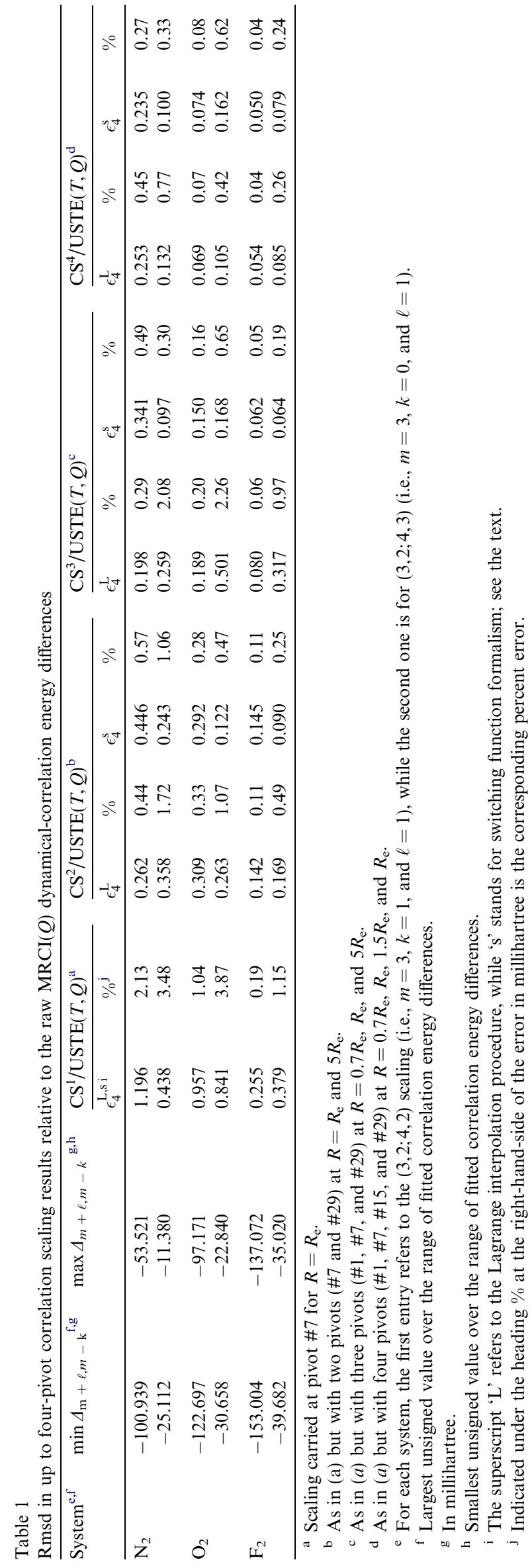


CAS energy $(X=6)$. Thus, we expect the $\operatorname{CS}^{N} / \operatorname{USTE}(T, Q)$ potentials to be rather accurate, with an accuracy most likely superior to the raw AV6Z energies. They are shown in Figs. 2-4 for the ground electronic states of $\mathrm{N}_{2}, \mathrm{O}_{2}$, and $\mathrm{F}_{2}$, with the calculated points (not shown) connected for clarity by cubic splines.

Also shown for $\mathrm{N}_{2}$ in Fig. 2 are the extrapolated CAS and raw $\operatorname{MRCI}(Q)$ correlation energies, as well as the turning points [21] obtained from the RKR inversion of spectroscopic data. The notable feature is the good agreement between the extrapolated curve and the empirical RKR points. Another visible trend from the insert of this figure is the more abrupt approach to dissociation with increasing cardinal number. This may be rationalized from the well known basis set superposition error (BSSE), which tends to make the potential more attractive for poor basis sets but vanishes for an infinite basis set. The shape of the potential manifests on the calculated vibrational term val-

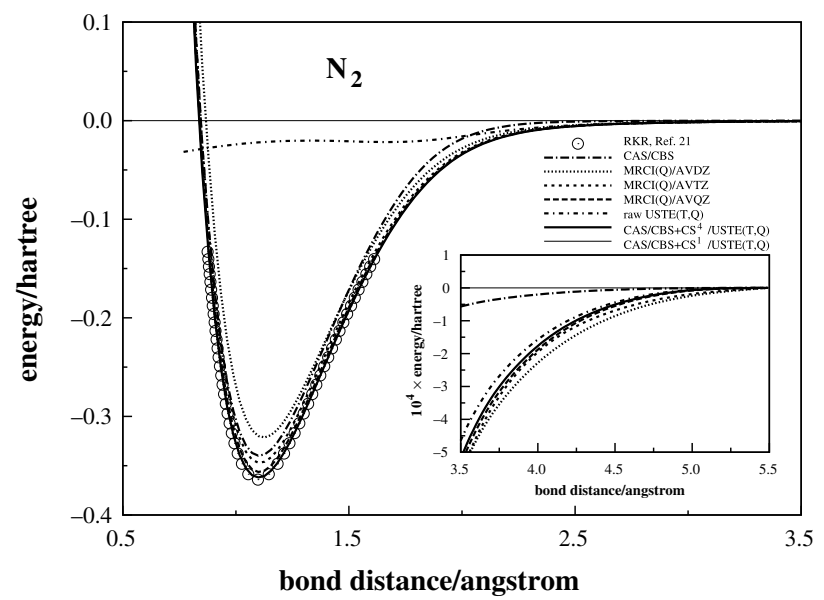

Fig. 2. Potential energy curves of $\mathrm{N}_{2}$. Shown are the $\mathrm{MRCI}(Q) / \mathrm{AV} X \mathrm{Z}$ and $\operatorname{CS}^{N} / \operatorname{USTE}(T, Q)(N=1,4)$ curves, in addition to the $\operatorname{USTE}(T, Q)$ dynamical correlation, CAS/CBS, and spectroscopic RKR energies. In this and subsequent plots, the CAS/CBS energy used to calculate the $\mathrm{CAS}^{N} / \mathrm{USTE}(T, Q)$ potentials has been obtained by using our own rule $[10]$ with $(T, Q, 5,6)$ raw energies; see also text.

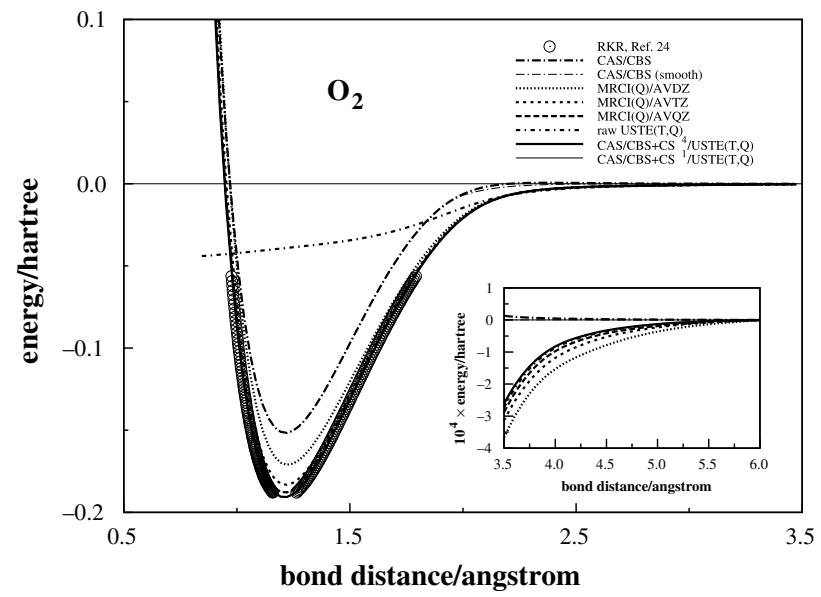

Fig. 3. As in Fig. 2 but for the ground electronic state of $\mathrm{O}_{2}$.

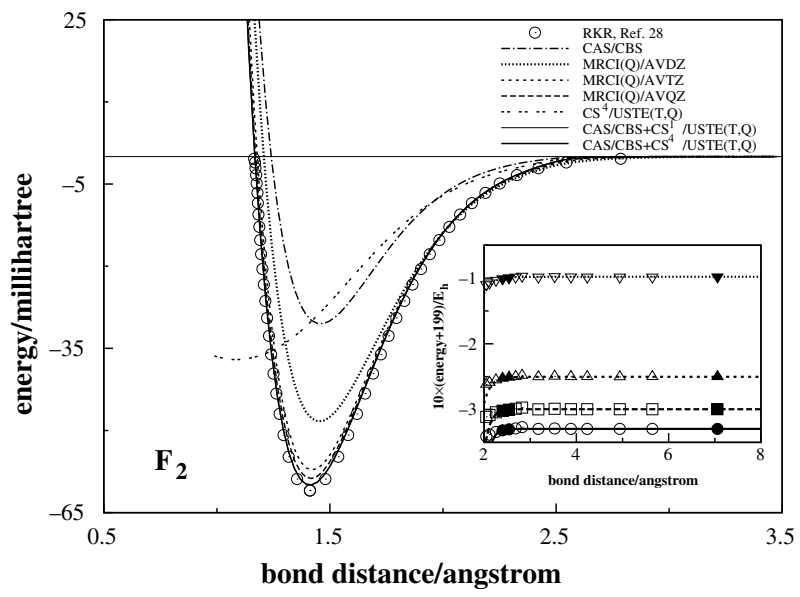

Fig. 4. As in Fig. 2, the main panel shows the potential energy curves of $\mathrm{F}_{2}$. Shown in the insert are the 'non-smoothed' ab initio energies (symbols), and the corresponding 'smoothed' curves (lines; see the text) which were fitted by reproducing the (three) solid symbols. These curves are valid only after the innermost fitted point, and should illustrate the 'roughness' (as manifested by the ups and downs relative to the dissociation asymptote) of the raw ab initio points at intermediate bond distances, although attenuated in the scale of the figure.

ues that are collected in Table 2. To reduce at a minimum any influence of fitting the calculated data to an analytic function, all ro-vibrational calculations here reported have been carried out on spline-fitted curves, suitably extrapolated to the asymptotes as implemented in Ref. [22]. Although the number of calculated raw energies may be too small for accurate spectroscopic calculations, it should suffice to warrant the trends here reported. As seen from Table 2, all $\operatorname{CS}^{N} / \operatorname{USTE}(T, Q)$ potentials reach $\operatorname{AV7Z}$ or even higher quality. Although basically equivalent when judged from their rmsd, it is interesting to note that $\mathrm{CS}^{1} /$ $\operatorname{USTE}(T, Q)$ yields typically as accurate results as the more elaborate $\mathrm{CS}^{N \geqslant 2} / \operatorname{USTE}(T, Q)$ ones. This may not be surprising since limited $\mathrm{CI}$ methods such as $\operatorname{MRCI}(Q)$ are well known to suffer from size-extensivity errors [23], and hence such deficiencies may extend to the $\operatorname{CS}^{N} / \operatorname{USTE}(T, Q)$ energies. We emphasize though that only $\operatorname{MRCI}(Q)$ calculations using the modest $\mathrm{AVDZ}$ and AVTZ basis sets, and 1 to 4 points using $\mathrm{AVQZ}$, have been performed.

The potential curves for the ground electronic state of molecular oxygen are depicted in Fig. 3. Besides some trends that are akin to $\mathrm{N}_{2}$, the striking feature is perhaps the occurrence of a small barrier in the CAS/CBS curve (this result is common to all raw CAS/AVXZ curves), a fact that is also very much present in the case of $F_{2}$ to be discussed later. Since this barrier is small and does not show up in the $\operatorname{MRCI}(Q) / \operatorname{AV} X \mathrm{Z}$ curves, we have ignored it at first. The calculated vibrational term values are gathered in Table 2. Interestingly, the $\operatorname{CS}^{1} / \operatorname{USTE}(T, Q)$ potential gives results as good as $\operatorname{CS}^{N \geqslant 2} / \operatorname{USTE}(T, Q)$ when judged from the RKR turning points [24]. Somewhat surprisingly, we observe only a very small improvement when going from the raw AV5Z to AV6Z terms values. Although this might lead to conclude that the results were converged 
Table 2

Vibrational term values $G_{v}$ of $\mathrm{N}_{2}, \mathrm{O}_{2}$, and $\mathrm{F}_{2}$

\begin{tabular}{|c|c|c|c|c|c|c|c|c|c|c|c|}
\hline \multirow[t]{2}{*}{ System } & \multirow[t]{2}{*}{$v$} & \multicolumn{9}{|c|}{ Theoretical $^{\mathrm{a}}$} & \multirow[t]{2}{*}{ exp. ${ }^{f}$} \\
\hline & & $\mathrm{AVDZ}$ & $\mathrm{AVTZ}$ & $\mathrm{AVQZ}$ & AV5Z & AV6Z & $\mathrm{CS}^{1 \mathrm{~b}}$ & $\mathrm{CS}^{2 \mathrm{c}}$ & $\mathrm{CS}^{3 \mathrm{~d}}$ & $\mathrm{CS}^{4 \mathrm{e}}$ & \\
\hline \multirow[t]{10}{*}{$\mathrm{N}_{2}$} & 0 & 29.6 & 17.1 & 9.4 & 6.8 & 6.0 & 4.4 & 4.8 & 4.2 & 4.1 & 1175.8 \\
\hline & 1 & 89.0 & 51.3 & 27.9 & 20.1 & 17.7 & 12.7 & 13.9 & 12.4 & 12.2 & 3505.7 \\
\hline & 2 & 149.3 & 85.4 & 46.3 & 33.3 & 29.3 & 20.9 & 22.9 & 20.7 & 20.4 & 5806.9 \\
\hline & 3 & 210.7 & 119.7 & 64.6 & 46.6 & 40.9 & 29.1 & 31.8 & 29.1 & 28.7 & 8079.5 \\
\hline & 4 & 273.1 & 154.1 & 82.8 & 59.7 & 52.2 & 37.1 & 40.5 & 37.4 & 36.9 & 10323.3 \\
\hline & 5 & 336.5 & 188.7 & 100.9 & 72.8 & 63.5 & 45.0 & 49.1 & 45.6 & 45.0 & 12538.3 \\
\hline & 10 & 673.3 & 365.5 & 190.8 & 137.1 & 117.8 & 82.8 & 89.5 & 85.0 & 84.0 & 23180.2 \\
\hline & 15 & 1051.6 & 551.7 & 280.3 & 199.6 & 169.1 & 117.5 & 125.6 & 120.8 & 119.6 & 33094.4 \\
\hline & $\mathrm{rmsd}^{\mathrm{g}}$ & 1032.5 & 526.5 & 263.3 & 186.0 & 156.5 & 107.7 & 114.7 & 110.7 & 109.7 & \\
\hline & & & & & & & 102.6 & 109.6 & 105.5 & 104.5 & \\
\hline \multirow[t]{10}{*}{$\mathrm{O}_{2}$} & 0 & 22.4 & 15.0 & 4.1 & 2.2 & 2.2 & 0.0 & -0.1 & -0.4 & -0.3 & 787.4 \\
\hline & 1 & 68.6 & 44.5 & 12.0 & 6.1 & 6.0 & -0.7 & -1.0 & -1.7 & -1.3 & 2343.8 \\
\hline & 2 & 117.1 & 73.3 & 19.7 & 9.7 & 9.6 & -1.7 & -2.3 & -3.2 & -2.5 & 3876.6 \\
\hline & 3 & 167.5 & 101.4 & 27.3 & 13.0 & 12.9 & -2.9 & -3.8 & -4.9 & -4.0 & 5386.0 \\
\hline & 4 & 219.8 & 128.9 & 34.6 & 16.1 & 16.0 & -4.3 & -5.6 & -6.9 & -5.6 & 6872.3 \\
\hline & 5 & 273.8 & 155.7 & 41.8 & 19.0 & 18.9 & -6.1 & -7.6 & -9.1 & -7.4 & 8335.6 \\
\hline & 10 & 564.3 & 280.0 & 75.7 & 30.7 & 30.2 & -17.9 & -21.1 & -22.9 & -19.4 & 15310.9 \\
\hline & 15 & 882.6 & 390.8 & 107.4 & 39.0 & 35.5 & -36.0 & -40.9 & -42.7 & -37.5 & 21718.2 \\
\hline & $\mathrm{rmsd}^{\mathrm{g}}$ & 772.3 & 332.4 & 91.2 & 32.1 & 29.1 & 34.0 & 38.0 & 39.4 & 35.1 & \\
\hline & & & & & & & 51.1 & 55.2 & 56.7 & 52.3 & \\
\hline \multirow{10}{*}{$\mathrm{F}_{2}$} & 0 & 53.8 & 8.3 & 5.8 & 3.0 & 2.1 & 1.2 & 1.0 & 0.9 & 0.9 & 458.3 \\
\hline & 1 & 156.3 & 20.5 & 12.1 & 3.9 & 1.0 & -1.6 & -2.2 & -2.5 & -2.4 & 1352.2 \\
\hline & 2 & 259.9 & 34.6 & 19.3 & 5.7 & 0.9 & -3.5 & -4.5 & -5.0 & -4.7 & 2222.5 \\
\hline & 3 & 364.7 & 50.4 & 27.4 & 8.6 & 1.8 & -4.5 & -6.1 & -6.7 & -6.2 & 3068.5 \\
\hline & 4 & 470.8 & 68.2 & 36.6 & 12.7 & 4.0 & -4.4 & -6.6 & -7.3 & -6.6 & 3889.8 \\
\hline & 5 & 578.5 & 88.2 & 47.1 & 18.3 & 7.6 & -3.0 & -5.9 & -6.6 & -5.7 & 4685.8 \\
\hline & 10 & 1138.1 & 219.4 & 119.6 & 67.6 & 47.1 & 22.5 & 15.6 & 14.9 & 17.1 & 8256.8 \\
\hline & 15 & 1729.2 & 410.3 & 231.7 & 157.1 & 126.4 & 81.7 & 70.2 & 69.6 & 72.6 & 11057.9 \\
\hline & $\mathrm{rmsd}^{\mathrm{g}}$ & 1428.0 & 374.1 & 206.7 & 134.3 & 109.8 & 71.5 & 62.6 & 62.3 & 64.4 & \\
\hline & & & & & & & 86.3 & 72.9 & 72.5 & 78.2 & \\
\hline
\end{tabular}

${ }^{\text {a }}$ Deviations, $G_{v}^{\text {exp }}-G_{v}^{\text {calc }}$. Under the headings $\operatorname{AV} X \mathrm{Z}$ are the results obtained for the raw $\mathrm{MRCI}(Q) / \mathrm{AV} X \mathrm{Z}$ calculations.

b Based on the $\mathrm{CS}^{1} / \mathrm{USTE}(3,4)$ method from the present work.

c As in (a) but for $\mathrm{CS}^{2} / \mathrm{USTE}(T, Q)$.

${ }^{\mathrm{d}}$ As in (a) but for $\mathrm{CS}^{3} / \mathrm{USTE}(T, Q)$.

${ }^{\text {e }}$ As in (a) but for $\operatorname{CS}^{4} / \operatorname{USTE}(T, Q)$.

${ }^{\mathrm{f}}$ The zero-point energy has been taken from Huber and Herzberg [20], and added to the $G(v)$ values reported in Refs. [21,24] for $\mathrm{N}_{2}$, and $\mathrm{O}_{2}$, respectively. See Ref. [28] for $F_{2}$.

${ }^{g}$ Rmsd with respect to the experimental term values: $v \leqslant 24$ for $\mathrm{N}_{2} ; v \leqslant 22$ for $\mathrm{O}_{2} ; v \leqslant 22$ for $\mathrm{F}_{2}$. The first entry refers to the actual tabulated results, which employed CAS energies extrapolated from our own rule [10], i.e., using up to $X=6$ raw energies, while the $(T, Q)$ two-parameter rule of Karton and Martin [13] has been utilized for the second entry; see the text.

to within a few $\mathrm{cm}^{-1}$ at $X=6$, this is clearly not the case when judging from the empirical RKR data. Besides, we should have in mind that only the first 23 vibrational levels have been counted for the statistics. We recall that a direct least-squares fit [25] to 90 ro-vibrational spectroscopic term values [26] and the 23 RKR points reported by Krupenie [24], let to an $\mathrm{O}_{2}$ potential that reproduces the input data with a rmsd of $0.6 \mathrm{~cm}^{-1}$ for the 90 ro-vibrational energies $\left(1.4 \mathrm{~cm}^{-1}\right.$ with respect to the 18 vibrational energies), and $22 \mathrm{~cm}^{-1}$ for the RKR points. Somewhat coincidentally, perhaps, we obtain a rmsd of similar order of magnitude from our $\operatorname{CS}^{N} / \operatorname{USTE}(T, Q)$ curves. Table 3 gathers the rotational constants of $\mathrm{O}_{2}$ for vibrational states up to $v=21$. Despite typical deviations with respect to experiment of only $0.005 \mathrm{~cm}^{-1}$ (they are typically smaller by $10^{-3} \mathrm{~cm}^{-1}$ for the potentials based on the CAS/CBS energies from the two-parameter $(T, Q)$ rule of Karton and
Martin [13]; see later), the results obtained from all $\mathrm{CS}^{N} /$ $\operatorname{USTE}(T, Q)$ potentials are better than the raw $\operatorname{MRCI}(Q) /$ AV5Z and AV6Z ones. A final remark to note that removing the barrier in the CAS/CBS curve (thin dash-dot line in Fig. 3) has no noticeable effect in the vibrational energies.

The calculated curves for molecular fluorine are plotted in Fig. 4. As shown in the insert of this figure, all CAS and $\operatorname{MRCI}(Q) / \operatorname{AV} X \mathrm{Z} \quad(X=D, T, Q, 5,6) \quad$ calculations show instabilities beyond a reduced bond distance of about $R /$ $R_{\mathrm{e}}=2.5$, i.e., $R=3.53 \AA$. In an attempt to investigate whether this could be due to the step size used for the calculations, we have performed a dense grid calculation using the AVDZ basis set. No changes have been observed. We have also carried out two-state averaged CAS/AVDZ calculations in an attempt to explore whether such instabilities could be assigned to a nearby perturbing state of the same symmetry. At this level of theory, the first excited state 
Table 3

Rotational constants for the various vibrational states of $\mathrm{O}_{2}$ in $\mathrm{cm}^{-1}$

\begin{tabular}{|c|c|c|c|c|c|c|c|c|c|c|c|c|c|c|c|}
\hline \multirow[t]{2}{*}{$v$} & \multicolumn{7}{|c|}{ Theoretical $^{\mathrm{a}}$} & \multicolumn{8}{|c|}{ Experimental } \\
\hline & $\mathrm{AVDZ}$ & $\mathrm{AVTZ}$ & $\mathrm{AV} Q \mathrm{Z}$ & AV5Z & AV6Z & $\mathrm{CS}^{1}$ & $\mathrm{CS}^{4}$ & $\begin{array}{l}\text { Ref. } \\
{[26]}\end{array}$ & $\begin{array}{l}\text { Ref. } \\
\text { [29] }\end{array}$ & $\begin{array}{l}\text { Ref. } \\
{[30]}\end{array}$ & $\begin{array}{l}\text { Ref. } \\
\text { [31] }\end{array}$ & $\begin{array}{l}\text { Ref. } \\
{[32]}\end{array}$ & $\begin{array}{l}\text { Ref. } \\
\text { [33] }\end{array}$ & $\begin{array}{l}\text { Ref. } \\
{[34]}\end{array}$ & $\begin{array}{l}\text { Ref. } \\
{[35]}\end{array}$ \\
\hline 1 & 1.3805 & 1.3996 & 1.4116 & 1.4142 & 1.4142 & 1.4170 & 1.4169 & 1.4218 & & 1.4219 & & & & 1.4220 & \\
\hline 2 & 1.3639 & 1.3840 & 1.3960 & 1.3986 & 1.3986 & 1.4014 & 1.4013 & 1.4060 & & 1.4061 & & & 1.4068 & & \\
\hline 3 & 1.3474 & 1.3685 & 1.3805 & 1.3831 & 1.3831 & 1.3859 & 1.3858 & 1.3904 & & 1.3904 & & & 1.3886 & & \\
\hline 4 & 1.3311 & 1.3530 & 1.3650 & 1.3676 & 1.3676 & 1.3704 & 1.3703 & 1.3747 & & 1.3747 & & 1.3736 & 1.3742 & & \\
\hline 7 & 1.2824 & 1.3071 & 1.3188 & 1.3215 & 1.3215 & 1.3243 & 1.3242 & & & & & 1.3262 & & & \\
\hline 8 & 1.2663 & 1.2919 & 1.3035 & 1.3063 & 1.3062 & 1.3090 & 1.3089 & & & & & & & & \\
\hline 9 & 1.2502 & 1.2767 & 1.2882 & 1.2910 & 1.2909 & 1.2937 & 1.2936 & & & & 1.2978 & & & & \\
\hline 10 & 1.2342 & 1.2614 & 1.2729 & 1.2758 & 1.2756 & 1.2784 & 1.2784 & & & & 1.2823 & & & & \\
\hline 11 & 1.2181 & 1.2462 & 1.2576 & 1.2605 & 1.2603 & 1.2631 & 1.2631 & & & & 1.2659 & & & & 1.26 \\
\hline 12 & 1.2020 & 1.2309 & 1.2424 & 1.2453 & 1.2450 & 1.2478 & 1.2477 & & 1.2506 & & & & & & 1.250 \\
\hline 17 & 1.1199 & 1.1529 & 1.1648 & 1.1679 & 1.1670 & 1.1700 & 1.1699 & & 1.1717 & & & & & & 1.171 \\
\hline 18 & 1.1029 & 1.1367 & 1.1487 & 1.1518 & 1.1510 & 1.1540 & 1.1539 & & 1.1557 & & & & & & 1.156 \\
\hline 19 & 1.0856 & 1.1203 & 1.1322 & 1.1354 & 1.1348 & 1.1378 & 1.1377 & & & & & & & & 1.139 \\
\hline 20 & 1.0678 & 1.1036 & 1.1155 & 1.1187 & 1.1183 & 1.1213 & 1.1213 & & & & & & & & 1.121 \\
\hline 21 & 1.0495 & 1.0865 & 1.0984 & 1.1017 & 1.1014 & 1.1046 & 1.1045 & & & & & & & & 1.104 \\
\hline
\end{tabular}

${ }^{\text {a }}$ Notation as in Table 2.

appears well enough separated, and hence can hardly be responsible for such a disturbance. Moreover, the lower adiabatic curve continued to reveal a similar pattern, with a small bump appearing at moderate bond distances. To overcome the difficulty (which, as a matter of fact, can hardly be remedied in direct dynamics calculations using a one-state calculation), we have first attempted to fit both the raw $\operatorname{MRCI}(Q)$ and $\operatorname{CS}^{N} / \operatorname{USTE}(T, Q)$ curves using the dispersion-type form $V(R)=a+b / R^{6}+c / R^{8}$, where $a$ is fixed at the corresponding asymptotic energy (taken as $R \sim 7 \AA$ ), and $b$ and $c$ are parameters determined by, say, reproducing the two far most geometries prior to the disruption of the potential curve $(R=2.40$ and $2.54 \AA)$. Unfortunately, no solution free from artifacts (this would basically require both $b$ and $c$ to be negative such as to avoid developing a maximum before dissociation; $a$ itself is also negative when one considers the total electronic energy) could be found. We then turned to the exponential form $V(R)=a+b \exp (c R)$, with the parameters fixed as suggested above. The same procedure has been used to fix the CAS/CBS curve. The results are shown in Fig. 4, while the calculated vibrational term values are in Table 2. As in the cases of $\mathrm{N}_{2}$ and $\mathrm{O}_{2}$, the results are of high accuracy when compared with the empirical data. We observe that most vibrational levels that would be obtained in a more pragmatic way by ignoring outliers (the points that violate the form of a smooth potential curve) do not differ significantly from those here reported.

Some further remarks on the accuracy of the present scheme may be extracted from Fig. 5, which compares the results obtained from the present approach with those

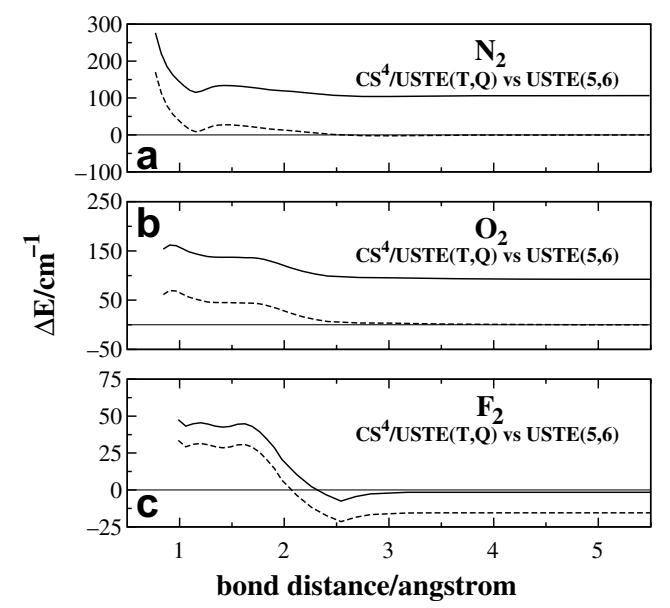

Fig. 5. Difference between the potentials obtained by extrapolating the dynamical correlation at the two highest affordable cardinal numbers, USTE $(5,6)$, and the $\operatorname{CS}^{4} / \operatorname{USTE}(3,4)$ ones: (a) $\mathrm{N}_{2}$; (b) $\mathrm{O}_{2}$; (c) $\mathrm{F}_{2}$. The dashed lines are the deviations in relative energy (with respect to the asymptote), and the solid lines in absolute energy.

obtained by utilizing dynamical correlation energies scaled pointwise from $\operatorname{MRCI}(Q)$ calculations with the two large affordable basis sets, namely using $\operatorname{USTE}(5,6)$. First, we observe that except for short distances in the repulsive wing of the potential curve, the absolute error seldom reaches an unsigned value larger than $125 \mathrm{~cm}^{-1}$ for both $\mathrm{N}_{2}$ and $\mathrm{O}_{2}$, and $40 \mathrm{~cm}^{-1}$ for $F_{2}$. When looking at relative energies, such values reduce in the above order to 50 and $25 \mathrm{~cm}^{-1}$.

Table 4 gathers the calculated equilibrium geometries as obtained from a parabolic fit to the three points with 
Table 4

Deviations of raw and extrapolated equilibrium geometries ${ }^{\mathrm{a}}$ and dissociation energies ${ }^{\mathrm{b}}$ of $\mathrm{N}_{2}, \mathrm{O}_{2}$, and $\mathrm{F}_{2}$ with respect to the experimental values ${ }^{\mathrm{c}, \mathrm{d}}$

\begin{tabular}{|c|c|c|c|c|c|c|c|c|c|c|c|c|c|c|c|c|}
\hline \multirow[t]{2}{*}{ System/time } & \multicolumn{2}{|c|}{$\mathrm{AVDZ}$} & \multicolumn{2}{|c|}{$\mathrm{AVTZ}$} & \multicolumn{2}{|c|}{$\mathrm{AVQZ}$} & \multicolumn{2}{|l|}{$\mathrm{CS}^{1 \mathrm{e}}$} & \multicolumn{2}{|l|}{$\mathrm{CS}^{2 \mathrm{e}}$} & \multicolumn{2}{|l|}{$\mathrm{CS}^{3 \mathrm{e}}$} & \multicolumn{2}{|l|}{$\mathrm{CS}^{4 \mathrm{e}}$} & \multicolumn{2}{|l|}{ exp..$^{f}$} \\
\hline & $\Delta R_{\mathrm{e}}$ & $\Delta D_{\mathrm{e}}$ & $\Delta R_{\mathrm{e}}$ & $\Delta D_{\mathrm{e}}$ & $\Delta R_{\mathrm{e}}$ & $\Delta D_{\mathrm{e}}$ & $\Delta R_{\mathrm{e}}$ & $\Delta D_{\mathrm{e}}$ & $\Delta R_{\mathrm{e}}$ & $\Delta D_{\mathrm{e}}$ & $\Delta R_{\mathrm{e}}$ & $\Delta D_{\mathrm{e}}$ & $\Delta R_{\mathrm{e}}$ & $\Delta D_{\mathrm{e}}$ & $R_{\mathrm{e}}$ & $D_{\mathrm{e}}$ \\
\hline $\mathrm{N}_{2}$ & 0.026 & 42.28 & 0.011 & 16.72 & 0.008 & 7.57 & $\begin{array}{l}0.006 \\
0.006\end{array}$ & $\begin{array}{l}2.01 \\
1.66\end{array}$ & $\begin{array}{l}0.006 \\
0.006\end{array}$ & $\begin{array}{l}2.17 \\
1.71\end{array}$ & $\begin{array}{l}0.006 \\
0.006\end{array}$ & $\begin{array}{l}2.17 \\
1.71\end{array}$ & $\begin{array}{l}0.006 \\
0.006\end{array}$ & $\begin{array}{l}2.17 \\
1.71\end{array}$ & 1.09768 & -364.046 \\
\hline CPU time ${ }^{\mathrm{g}}$ & \multicolumn{2}{|c|}{0.30} & \multicolumn{2}{|c|}{0.60} & \multicolumn{2}{|c|}{1.99} & & & & & & & & & & \\
\hline $\mathrm{O}_{2}$ & 0.020 & 20.29 & 0.013 & 8.22 & 0.009 & 3.48 & $\begin{array}{l}0.007 \\
0.006\end{array}$ & $\begin{array}{l}0.74 \\
0.52\end{array}$ & $\begin{array}{l}0.007 \\
0.006\end{array}$ & $\begin{array}{l}0.69 \\
0.46\end{array}$ & $\begin{array}{l}0.007 \\
0.006\end{array}$ & $\begin{array}{l}0.69 \\
0.46\end{array}$ & $\begin{array}{l}0.007 \\
0.006\end{array}$ & $\begin{array}{l}0.68 \\
0.46\end{array}$ & 1.20752 & -191.540 \\
\hline CPU time ${ }^{\mathrm{g}}$ & \multicolumn{2}{|c|}{0.22} & \multicolumn{2}{|c|}{0.44} & \multicolumn{2}{|c|}{1.59} & & & & & & & & & & \\
\hline $\mathrm{F}_{2}$ & 0.041 & 14.15 & 0.014 & 5.38 & 0.009 & 3.85 & $\begin{array}{l}0.006 \\
0.006\end{array}$ & $\begin{array}{l}2.76 \\
2.79\end{array}$ & $\begin{array}{l}0.006 \\
0.006\end{array}$ & $\begin{array}{l}2.67 \\
2.69\end{array}$ & $\begin{array}{l}0.006 \\
0.006\end{array}$ & $\begin{array}{l}2.67 \\
2.69\end{array}$ & $\begin{array}{l}0.006 \\
0.006\end{array}$ & $\begin{array}{l}2.67 \\
2.69\end{array}$ & 1.41193 & -62.663 \\
\hline CPU time $\mathrm{g}$ & \multicolumn{2}{|c|}{0.03} & \multicolumn{2}{|c|}{0.17} & \multicolumn{2}{|c|}{1.13} & & & & & & & & & & \\
\hline
\end{tabular}

Also indicated are the CPU times for a MRCI/AVXZ calculation at the equilibrium geometry (pivot \#1).

a $\Delta R_{\mathrm{e}}=R_{\mathrm{e}}^{\mathrm{calc}}-R_{\mathrm{e}}^{\mathrm{exp}}$, where the superscripts 'calc' and 'exp' stand in an obvious correspondence for calculated and experimental.

${ }^{\mathrm{b}} \Delta D_{\mathrm{e}}=D_{\mathrm{e}}^{\mathrm{calc}}-D_{\mathrm{e}}^{\text {exp }}$.

c Bond distances are in angstrom, energies in millihartree, and times in minutes.

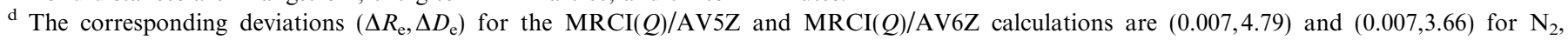
$(0.008,2.23)$ and $(0.007,1.64)$ for $\mathrm{O}_{2}$, and $(0.007,3.39)$ and $(0.007,3.17)$ for $\mathrm{F}_{2}$.

e Notation of theoretical methods as in previous Tables.

${ }^{\mathrm{f}}$ Refs. [20,28].

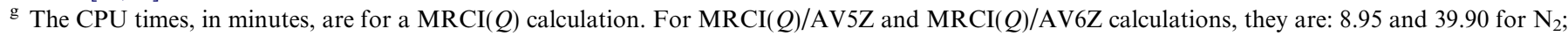

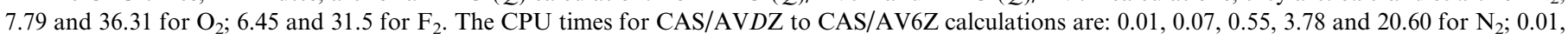
$0.07,0.55,3.81$ and 20.80 for $\mathrm{O}_{2} ; 0.01,0.07,0.57,3.72$ and 20.65 for $\mathrm{F}_{2}$.

lowest energy. Note that by choosing pivot \#1 to coincide with the experimental equilibrium geometry by no means implies that the latter will coincide with the minimum of the extrapolated curves. This is seen to exceed experiment by $0.006-0.007 \AA$ while the well depths are $0.7-2.2 \mathrm{mE}_{\mathrm{h}}$ $\left(0.4-1.4 \mathrm{kcal} \mathrm{mol}^{-1}\right)$ too shallow when compared with experiment [20]. Our results are found to compare well with explicitly correlated MR-CI estimates (often corrected for the basis set superposition error): e.g., Ref. [27] for $\mathrm{N}_{2}$ which also gives references to earlier work. One should recall at this stage that we have ignored core-correlation effects by considering only $10(2 \mathrm{~s}$ and $2 \mathrm{p}$ ) correlated electrons. Additionally, we could not account for the inescapable intrinsic error of the wave function model or the corrections due to relativistic effects and breakdown of the Born-Oppenheimer approximation. One hopes, that consideration of such effects will enhance the predictive capabilities of our scheme.

A final issue concerns the cost of the present approach when used to calculate a PES. Its efficiency with respect to a scheme based on the pointwise extrapolation from $\operatorname{MRCI}(Q) / \operatorname{AV} 5 Z$ and $\operatorname{MRCI}(Q) / \operatorname{AV} 6 Z$ energies can be assessed by the ratio

$\eta=\frac{N_{\mathrm{p}}\left(\tau_{6}^{\mathrm{MRCI}(Q)}+\tau_{5}^{\mathrm{MRCI}(Q)}\right)}{N_{\mathrm{p}}\left(\tau_{D}^{\mathrm{MRCI}(Q)}+\tau_{T}^{\mathrm{MRCI}(Q)}+\tau_{5}^{\mathrm{CAS}}+\tau_{6}^{\mathrm{CAS}}\right)+N \tau_{Q}^{\mathrm{MRCI}(Q)}+\left(N_{\mathrm{p}}-N\right) \tau_{Q}^{\mathrm{CAS}}}$

where $N_{\mathrm{p}}$ and $N$ have the meaning assigned in Section 1, and $\tau_{X}$ are the timings of the various calculations (indicated as superscript). Typical CPU times obtained from a single calculation at the geometry of pivot \#7 are gathered in Table 4 . Because the CAS/AV5Z and CAS/AV6Z calculations (including the ROHF cycle [19]) are rather expensive, the above ratio is $\eta \sim 2$. An obvious way to further enhance the efficiency of our method is by using an extrapolation scheme for the CAS energy that employs raw energies with cardinal numbers no larger than $X=Q$. For this, we have tested the two-parameter formula of Karton and Martin [13]. Although still yielding reasonable values, a generalization of our extrapolation rule [10] that uses such data seems to underestimate the best CAS/CBS energies, while a fit using Eq. (4) usually leads to an overestimation. As Table 4 shows, the $\left(R_{\mathrm{e}}, D_{\mathrm{e}}\right)$ attributes of the potentials so obtained are typically within the accuracy of a $\operatorname{MRCI}(Q) / \mathrm{AV} 7 \mathrm{Z}$ calculation or so when judged from the experimental data, although some differences occur in the spectroscopy of $\mathrm{O}_{2}$ for which we could not find a simple explanation. The $\eta$ ratios vary now from 29 for $\mathrm{N}_{2}$ with 4 pivots to over 48 for $\mathrm{F}_{2}$ with 1 pivot. Note that the CPU time savings are still large when comparing a $\operatorname{CS}^{1} / \operatorname{USTE}(T, Q)$ potential based on a $(T, Q)$ extrapolation of the CAS energy with a raw $\operatorname{MRCI}(Q) / A V 5 Z$ calculation. The ratios are in this case of $\eta=6.0,6.3$, and 6.2 for $\mathrm{N}_{2}, \mathrm{O}_{2}$, and $\mathrm{F}_{2}$, respectively. In summary, the $\operatorname{CS}^{N} / \operatorname{USTE}(T, Q)$ potentials here reported can achieve a performance only matched by $\operatorname{MRCI}(Q) /$ AV6Z or even higher quality potentials, but at a much lower computational cost. This, added to the fact that AVQZ basis sets are often just affordable for many systems of interest, makes the present scheme also promising in such cases.

\section{Conclusions}

We have generalized our previously reported scheme [15] that enables a potential energy surface corresponding 
to a calculation with a large basis set to be obtained from small basis sets calculations. The idea of scaling the correlation energy at a single pivot has accordingly been extended to include an arbitrary number of pivots. The application of the method to achieve the one-electron basis set limit has also been explored. Although the multi-pivot schemes have shown to yield curves that mimic the raw $a b$ initio curve calculated at the target basis set level better than $\mathrm{CS}^{1}$ by factors of fivefold or more, this by no means implies that the potentials so generated lead to a better agreement with the available spectroscopic data. This has partly been ascribed to the theoretical method utilized to calculate the raw data, namely the $\operatorname{MRCI}(Q)$ method which is known to suffer from size-extensivity errors. Irrespective of the number of pivotal geometries used for correlation scaling, the new methods suggest that highly accurate potentials can be obtained with modest basis sets that are often considered obsolete for quantitative purposes. This may lead to savings in computer time up to a factor of about 50 smaller than when attempting the direct solution of the non-relativistic electronic Schrödinger equation using $\operatorname{MRCI}(Q) / \mathrm{AV} X \mathrm{Z}$ methods. Although the method has been tested on diatomic curves, there is no reason of principle why it should not work for polyatomic molecules. For example, in the case of an A-BC interaction, the common strategy is to scan cuts for fixed values of the Jacobi angle, which very much resemble diatomic potentials. Of course, maxima may occur along such cuts as indeed it also happens in some diatomic systems, e.g., the $A^{1} \Pi$ state of CO. Work under way for this system indicates though that the method can yield equally successful results. Finally, the method may offer a promising route for accurate dynamics on-the-fly.

\section{Acknowledgements}

This work has been supported by Fundação para a Ciência e Tecnologia, Portugal (contracts POCI/QUI/ 60501/2004, POCI/AMB/60261/2004, and REEQ/128/ QUI/2005).

\section{References}

[1] T. Helgaker, P. Jørgensen, J. Olsen, Molecular Electronic-Structure Theory, Wiley, Chichester, 2000.

[2] H.-J. Werner, P.J. Knowles, J. Chem. Phys. 89 (1988) 5803.

[3] P.J. Knowles, H.-J. Werner, Chem. Phys. Lett. 145 (1988) 514.

[4] T.H. Dunning Jr., K.A. Petersson, D.E. Woon, in: P.v.R. Scleyer, N.L. Allinger, T. Clark, J. Gesteiger, P.A. Kolman, H.F. Schaefer
III (Eds.), Encyclopedia of Computational Chemistry, Wiley, Chichester, 1998 , p. 88.

[5] Basis sets were obtained from the Extensible Computational Chemistry Environment Basis Set Database, Version 02/25/04, as developed and distributed by the Molecular Science Computing Facility, Environmental and Molecular Sciences Laboratory which is part of the Pacific Northwest Laboratory, P.O. Box 999, Richland, Washington 99352, USA, and funded by the U.S. Department of Energy. The Pacific Northwest Laboratory is a multi-program laboratory operated by Battelle Memorial Institute for the U.S. Department of Energy under contract DE-AC06-76RLO 1830. Contact Karen Schuchardt for further information.

[6] T. Helgaker, W. Klopper, H. Koch, J. Noga, J. Chem. Phys. 106 (1997) 9639.

[7] D.G. Truhlar, Chem. Phys. Lett. 294 (1998) 45.

[8] A.J.C. Varandas, J. Chem. Phys. 113 (2000) 8880.

[9] W. Klopper, Mol. Phys. 6 (2001) 481.

[10] A.J.C. Varandas, J. Chem. Phys. 126 (2007) 244105.

[11] C. Schwartz, Phys. Rev. 126 (1962) 1015.

[12] W. Kutzelnigg, J.D. Morgan III, J. Chem. Phys. 96 (1992) 4484.

[13] A. Karton, J.M.L. Martin, Theor. Chim. Acta 115 (2006) 330.

[14] A.J.C. Varandas, Phys. Scr. (Comm. At. Opt. Mol. Phys.), in press.

[15] A.J.C. Varandas, P. Piecuch, Chem. Phys. Lett. 430 (2006) 448.

[16] F.B. Brown, D.G. Truhlar, Chem. Phys. Lett. 117 (1985) 307.

[17] A.J.C. Varandas, J. Chem. Phys. 90 (1989) 4379.

[18] P.L. Fast, J. Corchado, M.L. Sanchez, D.G. Truhlar, J. Phys. Chem. A 103 (1999) 3139.

[19] H.-J. Werner, et al., MOLPRO, version 2002.6, a package of ab initio programs, see www.molpro.net, 2003.

[20] K.P. Huber, G. Herzberg, Molecular Spectra and Molecular Structure IV. Constants of Diatomic Molecules, Van Nostrand, New York, 1979.

[21] S. Edwards, J. Roncin, F. Launay, F. Rostas, J. Mol. Spectrosc. 162 (1993) 257.

[22] R.J. LeRoy, LEVEL 7.5, A computer program for Solving the Radial Schrödinger Equation for Bound and Quasi-bound Levels, University of Waterloo Chemical Physics Research Report CP-655, 2002.

[23] W. Duch, G.H. Diercksen, J. Chem. Phys. 101 (1994) 3018.

[24] P.H. Krupenie, J. Phys. Chem. Ref. Data 1 (1972) 423.

[25] A.J.C. Varandas, S.P.J. Rodrigues, V.M.O. Batista, Chem. Phys. Lett. 424 (2006) 425.

[26] A.S. Cheung, K. Yoshino, J.R. Esmond, W.H. Parkinson, J. Mol. Spectrosc. 178 (1996) 66.

[27] R.J. Gdanitz, Chem. Phys. Lett. 283 (1998) 253.

[28] E.A. Colbourn, M. Dagenais, A.E. Douglas, J.W. Raymonda, Can. J. Phys. 54 (1976) 1343.

[29] C. Ciping, D.A. Ramsey, J. Mol. Spectrosc. 160 (1993) 512.

[30] G. Rouillé, G. Millot, R. Saint-Loup, H. Berger, J. Mol. Spectrosc. 154 (1992) 372.

[31] R.A. Copeland, P.C. Cosby, D.R. Crosley, J.B. Jeffries, T.G. Slanger, J. Chem. Phys. 86 (1987) 2500.

[32] V.N. Snopko, Opt. Spectrosc. 29 (1970) 445.

[33] M. Ogawa, Sci. Light 15 (1966) 97.

[34] H.D. Bacock, G. Herzberg, Astrophys. J. 108 (1948) 167.

[35] W. Lochte-Holtgreven, G.H. Dieke, Ann. Phys. 3 (1929) 937. 